\title{
Gravitational Lensing Described by Its Electromagnetic Processes
}

\author{
Hans W. Giertz \\ Uppsa Research, Gnesta, Sweden \\ Email: hans@miklagaard.com
}

Received 4 February 2014; revised 2 March 2014; accepted 10 March 2014

Copyright (C) 2014 by author and Scientific Research Publishing Inc.

This work is licensed under the Creative Commons Attribution International License (CC BY).

http://creativecommons.org/licenses/by/4.0/

(c) (i) Open Access

\begin{abstract}
In the present paper, gravitational lensing is described as the electromagnetic influence from gravity waves on light waves. Previous reports have described the dynamic electromagnetic processes of the atom, the photon and gravity. Results from these reports have been compiled into a theoretical model. The theoretical model describes the mechanism which results in gravitational lensing. The study also displays how the electromagnetic characteristics of gravity waves and light waves and the mechanism creating gravitational lensing are measured.
\end{abstract}

\section{Keywords}

\section{Gravitational Lensing; Atom; Gravity; Photon; Light Wave}

\section{Introduction}

It has been reported that the universe contains a gigantic singularity which generates very low frequency and synchronized TEM (transverse electromagnetic) waves, having extremely high amplitude and energy [1]-[3]. Atoms contain oscillators with different natural frequencies. These oscillators absorb TEM waves at specific frequencies. The absorbed electromagnetic energy accounts for atomic internal and external forces as well as atomic stored energy and emitted energy [1]-[3]. These TEM waves are impossible to measure using state of the art methods. However, a novel method enables exact characterization of TEM waves emitted by the singularity, absorbed by atoms and re-emitted by atoms [1]-[4].

It has also been reported that atoms absorb these TEM waves in the frequency range $0.005-0.03 \mathrm{~Hz}$ [1]. The absorbed energy is very large and may account for atomic forces and atomic stored energy.

Furthermore, it has been reported that atoms also absorb TEM waves with frequency $69.9 \mathrm{~Hz}$ [2]. These TEM waves are called gravity waves in the present paper. These gravity waves are then re-emitted uniformly into space, whereby some are absorbed by other atoms. This creates a mutual flow of synchronized TEM waves be- 
tween atoms, resulting in a mutual force of attraction, i.e. gravity.

It has also been reported that excess energy quanta $h v$ in oscillators, contained in a particle, an electron or an atom, is superpositioned on gravity waves absorbed by the oscillator. The re-emitted TEM wave has the superpositioned energy quanta $h v$ and the frequency $69.9 \mathrm{~Hz}+v$ [3]. The superpositioned energy quantum $h v$ is called photon and it explains the photon-wave duality. Empiric results reveal that this process is frequency independent [3] [4].

A large or compact body, e.g. a star, is characterized by its huge mass, i.e. its enormous amount of atoms [5] [6]. This implies that it re-emits vast amount of gravity waves. A light wave with frequency $69.9 \mathrm{~Hz}+v$, passing a compact body or a star, will experience a force. The mutual force of attraction between the light's $69.9 \mathrm{~Hz}$ component and gravity waves with frequency $69.9 \mathrm{~Hz}$, radiated from the body, results in a mutual force of attraction [2] which bends the light wave towards the body, i.e. gravitational lensing [7] [8].

The objective with the present paper is to build on results already presented in reports [1]-[4]. This information is compiled into a theoretical model in Section 2, which describes the mechanism behind gravitational lensing. The theoretical model is based on state of the art electromagnetic and wave theory [9]-[15], the Standard Model [16] and photon theory [17]-[20]. Discussion and conclusions are found in Section 3.

The aim of the study is to present a theoretical model of gravitational lensing.

\section{Theoretical Model}

\subsection{Gravity}

The theoretical model builds on the observation that atomic oscillators 69.9 , with natural frequency $69.9 \mathrm{~Hz}$, absorb TEM waves with frequency $69.9 \mathrm{~Hz}$ [2]. The TEM waves originate from a singularity in the universe and have been thoroughly described [1]-[3]. The absorbed TEM waves are then re-emitted uniformly into space.

The behavior and characteristics of plane TEM waves is independent of their frequency. The field vectors $\mathbf{E}$ and $\mathbf{B}$ or $\mathbf{H}\left(\mathbf{B}=\mu_{0} \mathbf{H}\right)$ are linked and perpendicular. The field vector amplitudes relate as $E=c B$, where $c$ is the speed of light. The electric and magnetic field vector amplitudes $E$ and $B$ decrease with the distance from the source as $1 / r$. The Poynting vector $\mathbf{S}$ describes the TEM energy flow density [9] [10]

$$
\mathbf{S}=\mathbf{E} \times \mathbf{H} \text {. }
$$

The energy stored in the magnetic field is just equal to that in the magnetic field. The direction of energy flow is reversed for a wave travelling in the opposite direction because the phase of $\mathbf{E}$ and $\mathbf{H}$ is reversed.

Assume that one source, Source 1, absorbs and re-emits plane TEM T $_{1}$ waves, described by its Poynting vector $\mathbf{S}_{1}(\mathrm{r})$ at the distance $r$ from Source 1 . Another source, Source 2 is positioned at the distance $r$. Source 2 absorbs TEM waves and re-emits plane $\mathrm{TEM}_{2}$ waves uniformly into space. In the direction towards Source 1 plane $\mathrm{TEM}_{1}$ and $\mathrm{TEM}_{2}$ waves interact because of the force between their synchronized field vectors. This also implies that there is energy transfer from $\mathrm{TEM}_{1}$ to $\mathrm{TEM}_{2}$ when $\mathrm{TEM}_{2}$ propagates towards Source 1 . In equilibrium there is energy transfer in both directions, i.e. from $\mathrm{TEM}_{1}$ to $\mathrm{TEM}_{2}$ vice versa. Note that $\mathbf{E}$ and $\mathbf{H}$ are always perpendicular and hence $\mathbf{S}=\mathbf{E} \times \mathbf{H}$ can be replaced by $S=E \cdot H$. Equilibrium is defined by the amount of energy that Source 1 is able to deliver at Source 2, e.g. at the distance $r, S_{1}(r)$, and the amount of energy that Source 2 is able to deliver at Source 1, i.e. $S_{2}(r)$. This results in back reaction force $F(r)$, i.e. radiation reaction [9]. This creates mutual force of attraction $F(r)$, and where $\gamma$ is a constant:

$$
F(r)=\gamma \cdot S_{1}(r) \cdot S_{2}(r) .
$$

It is now assumed that each source consists of many oscillators ${ }_{69.9}$ where each atomic oscillator og.9 $_{\text {re-emits }}$ TEM waves described by its Poynting vector $\delta S(r)$ and where $r$ denotes the distance from the source and its oscillator $_{69.9}$. $\mathbf{E}$ and $\mathbf{H}$ decrease linearly with the distance $r$ implying that $\delta S(r)$ decreases with the square of the distance; $\delta S(r)=\delta S(0) / r^{2}$. The mutual force of attraction $\delta F(r)$ between two atomic oscillators 69.9 with distance $r$ is

$$
\delta F(r)=\gamma \cdot \delta S \cdot \delta S / r^{2}
$$

It is now assumed that one oscillator $_{69.9}$ acts on $p$ collocated oscillators ${ }_{69.9}$, belonging to the set $\mathbf{P}$ of all oscillators $_{69.9}$ at Source 2, and where each force can be described by $\delta F(r)$. These oscillators 69.9 are synchronized resulting in the force 


$$
\sum_{p \in P} \delta F(r)=\gamma \cdot \delta S \cdot \sum_{p \in P} \delta S / r^{2}=\gamma \cdot(\delta S \cdot \delta S) \cdot p / r^{2} .
$$

It is now assumed that $n$ collocated oscillators ${ }_{69.9}$, belonging to the set $\mathbf{N}$ of all oscillators 69.9 at Source 1 , act on $p$ collocated oscillators 69.9 , belonging to the set $\mathbf{P}$ of all oscillators ${ }_{69.9}$ at Source 2, resulting in the total force $F(r)$

$$
F(r)=\gamma \cdot \sum_{n \in N} \delta S \cdot \sum_{p \in P} \delta S / r^{2}=\gamma \cdot(\delta S \cdot \delta S) \cdot n \cdot p / r^{2} .
$$

$\delta S \cdot \delta S$ is a constant described by the intrinsic characteristics of the oscillator ${ }_{69.9}$, and hence Equation (5) is simplified into

$$
F(r)=\xi \cdot n \cdot p / r^{2},
$$

where $\xi$ is a constant.

In a previous study Equation (6) was called the law of gravity between two clusters, at distance $r$ containing $n$ respectively $p$ oscillators $_{69.9}$ [2]. Note that the cluster can be few oscillators $_{69.9}$, an electron, an atom, a mass, a planet or a black hole.

Equation (6) can be illustrated with gravity between the earth and the sun. The earth contains $n$ atomic oscillators ${ }_{69.9}$ creating a force $n \cdot \delta F(r)$ on every atomic oscillator 69.9 in the sun. The sun contains $p$ oscillators $_{69.9}$, thus the total force of attraction is $n \cdot p \cdot \delta F(r)$ and that is equal to Equation (6). The earth's mass $m_{1}$ is proportional to the number of atomic oscillators 69.9 on earth, i.e. $m_{1} \sim n$, and the sun's mass $m_{2} \sim p$. Equation (6) is then approximately equal to the Newtonian geometric law of gravity, where $F_{G}$ is the gravitational force:

$$
F_{G}=\xi \cdot n \cdot p / r^{2} \approx F_{\text {Newton }}=G \cdot m_{1} \cdot m_{2} / r^{2} .
$$

$S_{1}(r)$ and $S_{2}(r)$ in Equation (6) depend on the distance between the singularity and $S_{1}(r)$ respectively $S_{2}(r)$. Thus $\xi$ in Equation (7) increases at decreasing distance to the singularity.

Gravity is illustrated in Figure 1.

\subsection{Photon}

The theory of photon energy transport in space can be illustrated with light waves. It has been shown [3] that light waves are the result of the sum of time limited processes, i.e. acceleration or deceleration of oscillators ${ }_{69.9}$ in an electron or atomic nucleus, see Figure 2. These oscillators ${ }_{69.9}$ absorb and re-emit gravity waves with frequency $69.9 \mathrm{~Hz}$. Accelerating or decelerating an electron or an atomic nucleus implies that they gain energy, i.e. each of their intrinsic oscillators ${ }_{69.9}$ increases its energy and this excess energy can be described by one or many

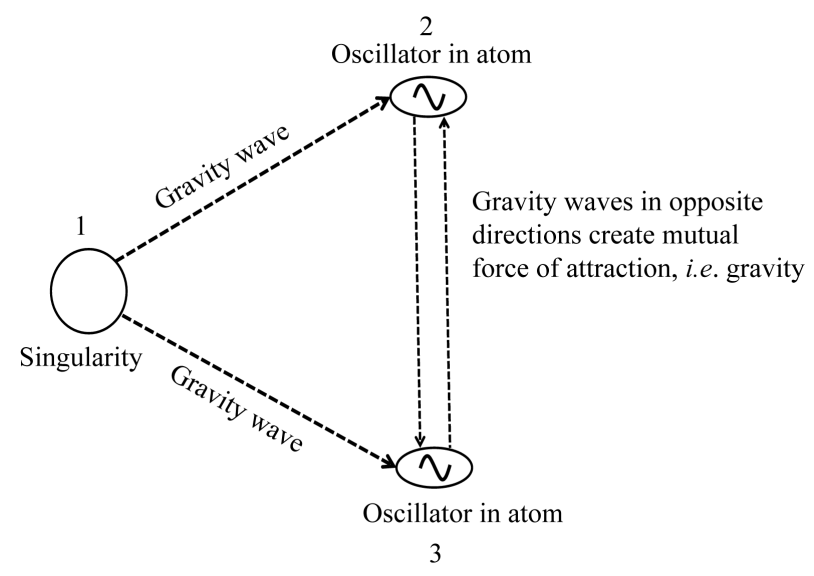

Figure 1. A singularity $\underline{1}$ generates gravity waves (low frequency plane TEM waves). Oscillators ${ }_{69.9} \underline{2}$ and $\underline{3}$ in atoms create resonance with gravity waves and re-emit them unaltered. This causes flow of gravity waves between atoms and their oscillators $69.9 \underline{2}$ and $\underline{3}$. The flow of gravity waves in opposite directions between oscillators $_{69.9}$ results in mutual force of attraction, i.e. gravity. 
4

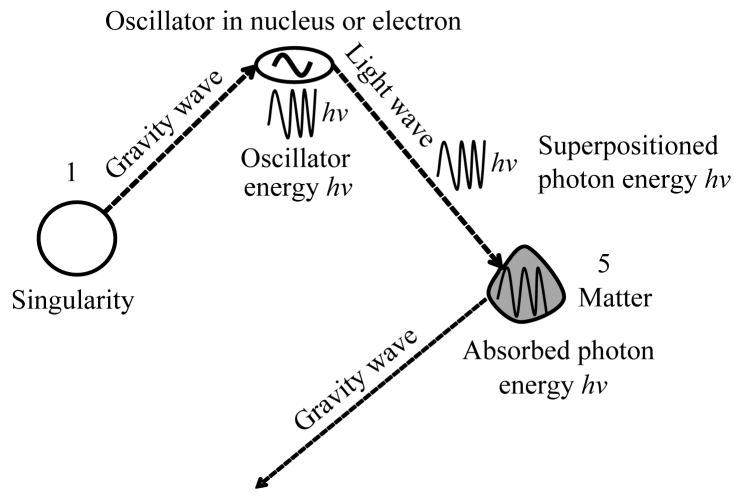

Figure 2. Electrons can be accelerated or decelerated. The electron contains oscillators 69.9 . The oscillator $69.9 \underline{4}$ in an electron creates resonance with a gravity wave and excess energy $h v$, caused by electron acceleration deceleration, is superpositioned on the re-emitted wave. The superpositioned energy $h v$ with frequency $v$ represents the photon. This wave with frequency $69.9 \mathrm{~Hz}$ and $v$ is re-emitted as a light wave. The superpositioned energy $h v$ can be absorbed in matter $\underline{5}$, whereby the light wave is converted into the original gravity wave with frequency $69.9 \mathrm{~Hz}$.

energy quanta $h v$. In this case the oscillator $69.9 \underline{4}$ in the electron or atom contains one energy quantum $h v$ at a time, where $h$ is Planck constant or quantum of action.

The energy quantum $h v$ is discrete and limited in time. The energy quantum $h v$ is superpositioned on a reemitted TEM wave. This TEM wave contains the superpositioned energy quantum $h v$ and the frequency $69.9 \mathrm{~Hz}$ and instant frequency $v$. Hence, the re-emitted wave is a gravity wave with superpositioned electromagnetic energy. The superpositioned electromagnetic energy quantum $h v$ with frequency $v$ is the photon. In a continuous process the sum of many photons and their energy quanta $h v$, superpositioned on re-emitted gravity waves, result in light waves.

The light wave's amplitude is proportional to the number of photons per time unit, which is proportional to the number of involved energy quanta $h v$, which in its turn is proportional to the number of involved oscillators $_{69.9}$ and which is proportional to the inertial mass. This light wave has the frequency $69.9 \mathrm{~Hz}+v$.

The light wave can be absorbed in matter $\underline{\mathbf{5}}$ and where the superpositioned energy quanta $h v$ are absorbed, resulting in that the light wave is converted back to the original gravity wave with frequency $69.9 \mathrm{~Hz}$.

The energy contained in a TEM wave is proportional to its frequency $v$. From this follows that the superpositioned energy and the photon energy $\delta E$ is:

$$
\delta E=\hbar \omega=h \nu .
$$

The TEM wave's superpositioned energy and momentum are related as $\delta E=p c$, where $p$ is the magnitude of the momentum vector $\mathbf{p}$. This derives from the following relativistic relation, with $m=0$ [11]:

$$
\begin{aligned}
& \delta E^{2}=p^{2} c^{2}+m^{2} c^{4}, \\
& \mathbf{p}=\hbar \mathbf{k}
\end{aligned}
$$

where $\mathbf{k}$ is the wave vector (where the wave number $k=|\mathbf{k}|=2 \pi v / c$ ), and $\hbar=h / 2 \pi$ is the reduced Planck constant. Since $\mathbf{p}$ points in the direction of the TEM wave propagation, the magnitude of the momentum is:

$$
p=\hbar k=h v / c .
$$

The photon also carries spin angular momentum that does not depend on its frequency $v$. The magnitude of its spin is $(2 \hbar)^{1 / 2}$ and the component measured along its direction of motion, its helicity, is $\pm \hbar$. These two possible helicities, called right-handed and left-handed, correspond to the two possible circular polarization states of the 
photon [16]-[18] and as described above.

Thus the photon contained in the light wave is not a particle; however, discrete electromagnetic energy quantum $h v$, with frequency $v$, superpositioned on a low frequency TEM wave, i.e. a gravity wave. From this follows that the photon is strictly mass less, has zero charge and propagates with the speed of the low frequency TEM wave, i.e. with the speed of light in free space.

\subsection{Gravitational Lensing}

The theoretical model encompasses a compact or large body like the sun, comprising enormous amount of atoms. It radiates vast amount of gravity waves (TEM waves) with frequency $69.9 \mathrm{~Hz}$ and where the energy flow density is described by the Poynting vector $\mathbf{S}$. Furthermore, the theoretical model comprises light, where the photon energy $h v$ is superpositioned on gravity waves with frequency $69.9 \mathrm{~Hz}$, resulting in a new TEM wave (i.e. the light wave) with the frequency $69.9 \mathrm{~Hz}+v$. The light wave energy is high due to its high frequency $v$; however, its low frequency component with frequency $69.9 \mathrm{~Hz}$ has low energy flow density and is described by its Poynting vector $\mathbf{S}$.

A light wave passing the sun can be described by the light wave's TEM wave and its $69.9 \mathrm{~Hz}$ component, where this component's energy flow density is described by the Poynting vector $\mathbf{S}_{1}$. Gravity waves, radiated from sun are described by their TEM waves with frequency $69.9 \mathrm{~Hz}$ and the Pointing vector $\mathbf{S}_{2}$. Synchronized TEM waves of equal frequency propagating in opposite direction or at an angel result in a mutual force of attraction where this force $\mathbf{F}$ is

$$
\mathbf{F}=\eta \cdot \mathbf{S}_{1} \cdot \mathbf{S}_{2},
$$

and where $\eta$ is a constant. The constant $\eta$ depends on the mass distribution of the large body and the light wave's energy, i.e. its frequency $v$.

This results in that the light wave is bent towards the gravity waves and towards the compact or large body, and which results in gravitational lensing [5]-[8].

The energy in the light wave is high due to its high frequency $v$ and $\mathbf{S}_{\mathbf{1}}$ is very small. Hence, $\mathbf{S}_{\mathbf{2}}$ must be very large in order to deflect the light wave. Consequently, gravitational lensing occurs primarily close to compact or large bodies. Gravitational lensing is illustrated in Figure 3.

\section{Discussion}

The strength of the present theory is that there exists a method to measure relevant parameters [1]-[4]. TEM waves originating from the singularity have been measured to a degree which allows exact description of their

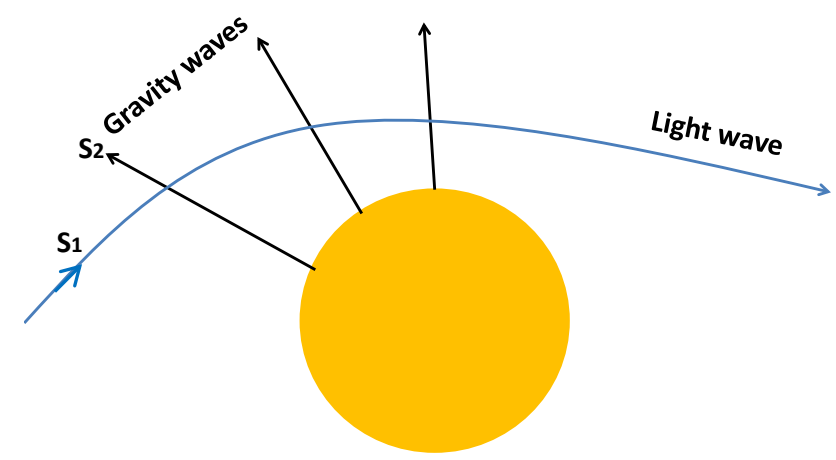

Figure 3. A light wave passing a compact or large body is bent towards the body, i.e. gravitational lensing. The light waves $69.9 \mathrm{~Hz}$ component's energy flow density is described by its Poynting vector $S_{1}$. The compact body radiates gravity waves with frequency $69.9 \mathrm{~Hz}$ and its energy flow density is $S_{2}$. This results in mutual force of attraction $\mathrm{F}$ between the waves: $\mathrm{F}=$ $\eta \cdot \mathrm{S}_{1} \cdot \mathrm{S}_{2}$, resulting in bending the light wave towards the gravity waves. The constant $\eta$ depends on the light wave frequency $v$ and the large body's mass distribution. 
characteristics such as direction of origin, amplitude, phase, frequencies and field vector behavior [1]-[3]. Furthermore, TEM waves, absorbed and re-emitted by atoms, have been measured to a degree which reveals the atomic intrinsic mechanism; forced damped oscillators with natural frequencies $0.005-0.03 \mathrm{~Hz}$ [1] and $69.9 \mathrm{~Hz}$ [2]. Moreover, extremely low frequency photons have been produced and measured in laboratory [3]. The force of attraction between TEM waves has been measured. Hence, crucial parts of the present theory have been measured in laboratory and some parts have been simulated and reproduced in laboratory.

It is proposed that all energy in the universe originates from a singularity, and is distributed throughout the universe by low frequency TEM waves, having a broad spectrum of frequencies. The atom in itself is fully passive. At this stage the atom's physical view is of little interest. However, the atom's functional view is crucial; the atom consist of forced damped oscillators with different natural frequencies. They absorb TEM waves originating from the singularity. The atomic oscillators are assembled in different configurations, and where the number of oscillators and their mix of natural frequencies result in different types of electromagnetic energies and forces, according to electromagnetic theory [5] [6] and wave theory [7]-[11]. One configuration results in that the absorbed energy creates internal forces (strong force) and nuclear binding energy [1]. A second configuration results in that the absorbed energy generates what is perceived as a static electric field, i.e. the Coulomb field [1]. A third configuration results in that absorbed energy is re-emitted creating gravity, photon, gravitational mass and inertial mass [2] [3]. All of these processes are entirely electromagnetic. These processes originate from external electromagnetic energy operating on fully passive atoms and where the atom contains only one type of functionality; forced damped oscillators.

The platform of modern physics is elementary particle physics described in the Standard Model [16]. Atom, photon and graviton are described as particles. The atom is self contained, i.e. atomic forces and energy are the consequence of atomic intrinsic mechanisms. The Higgs field is added in order to account for inertial mass.

\section{Conclusions}

The present study describes gravity, photon and gravitational lensing by their electromagnetic processes. It is a cumbersome task to explain these electromagnetic processes by means of (poorly defined) particles. Consequently, according to the Standard Model, the atom must contain some unknown phenomena which create force of attraction (i.e. strong force, nuclear binding energy, gravity). It also implies that there exists an intrinsic mechanism which can store enormous amount of nuclear energy forever, with no loss. It also implies that the atom can radiate energy, fields and particles (graviton) without losing energy or mass and without any change in its parameters. These are amazing properties. The consequence of the Standard Model is that the universe must contain a large number of different particles which supersede electromagnetism. However, the drawback of the Standard Model is that these particles and their relationship are poorly defined. The Standard Model falls short in explaining e.g. strong force, binding energy, photon, gravity, gravitational mass as well as a number of astrophysical phenomena (e.g. gravitational lensing, gravitational red \& blue shift, frame dragging, light conservation in black holes, expanding space).

The present paper describes gravitational lensing. Gravity is caused by TEM waves with frequency $69.9 \mathrm{~Hz}$. Light photons consist of energy quantum $h v$, superpositioned on gravity waves with frequency $69.9 \mathrm{~Hz}$. There is a mutual force of attraction between gravity waves and the light's gravity wave component. This force bends light waves towards the gravity wave, i.e. towards the compact or large body. The theoretical model is simplified. For instance gravity waves generated from a large body, like the sun, originate from different parts of the body, i.e. its mass distribution relative to the light wave. Close to the body Equation (5) and Equation (6) provide only approximate values. Close to the body Equation (12) and the Poynting vector $\mathbf{S}_{2}$ provide only approximate values. At close distance an exact Equation (12) must include the integral of $\mathbf{S}_{2}(r)$, where $r$ is the distance to different parts in the large body. Hence, a light wave passing close to a large body experiences a spectrum of forces according to Equation (12) which may produce multiple images. Likewise, the light wave's energy depends on its frequency $v$; hence, light waves containing a spectrum of frequencies $v$ result in multiple images. The paper does not elaborate on the difference between the strong and the weak gravitational lensing; however, it may be the result of the light wave's distance to the body and the body's size relative to this distance. The objective with the present paper is to describe the general principle of gravitational lensing. A complete model should include the influence from the light wave's spectrum, the body's mass distribution and the light wave's distance to the body. 


\section{References}

[1] Giertz, H.W. (2013) Atoms Absorb Low Frequency Electromagnetic Energy. Open Journal of Microphysics, 3, 115120. http://dx.doi.org/10.4236/ojm.2013.34017

[2] Giertz, H.W. (2013) Gravity Caused by TEM Waves Operating on Dipoles in Atoms. International Journal of Astronomy and Astrophysics, 3, 39-50. http://dx.doi.org/10.4236/ijaa.2013.32A007

[3] Giertz, H.W. (2013) Photons are EM Energy Superpositioned on TEM Waves. Open Journal of Microphysics, 3, 71-80. http://dx.doi.org/10.4236/ojm.2013.33013

[4] Giertz, H.W. (2010) Extremely Low Frequency Electromagnetic Energy in the Air. Journal of Atmospheric and SolarTerrestrial Physics, 72, 767-773. http://dx.doi.org/10.1016/j.jastp.2010.03.022

[5] Shapiro, S.L. and Teukolsky, S.A. (1983) Black Holes, White Dwarfs, and Neutron Stars: The Physics of Compact Objects. John Wiley and Sons, New York. http://dx.doi.org/10.1002/9783527617661

[6] Bozza, V. (2010) Gravitational Lensing by Black Holes. General Relativity and Gravitation, 42, 2269-2300. http://dx.doi.org/10.1007/s10714-010-0988-2

[7] Blandford, R.D. Narayan, R. (1992) Cosmological applications of gravitational lensing. Annual Review of Astronomy and Astrophysics, 30, 311-358. http://dx.doi.org/10.1146/annurev.aa.30.090192.001523

[8] Petters, A.O., Levine, H. and Wambsganss, J. (2001) Singularity Theory and Gravitational Lensing. Progress in Mathematical Physics, Birkhäuser, Basel. http://dx.doi.org/10.1007/978-1-4612-0145-8

[9] Melrose, D.B. and McPhedran, R.C. (1991) Electromagnetic Processes in Dispersive Media. Cambridge University Press, Cambridge. http://dx.doi.org/10.1017/CBO9780511600036

[10] Bleaney, B.I. and Bleaney, B. (1965) Electricity and Magnetism. Oxford University Press, Amen House, London.

[11] Kneubühl, F.K. (1997) Oscillations and waves. Springer, Berlin. http://dx.doi.org/10.1007/978-3-662-03468-2

[12] Serway, R.A. and Jewett, J.W. (2005) Principles of Physics. 4th Edition, Cengage Learning, Stamford.

[13] Ostrovsky, L.A. and Potapov, A.I. (2002) Modulated Waves: Theory and Application. Johns Hopkins University Press, Baltimore.

[14] Terras, A. (1999) Fourier Analysis on Finite Groups and Applications. Cambridge University Press, Cambridge.

[15] Alonso, M. and Finn, E.J. (1968) Fundamental University Physics Volume III: Quantum and Statistical Physics, Addison-Wesley.

[16] Burgess, C. and Moore, G. (2007) The Standard Model: A Primer. Cambridge University Press, Cambridge.

[17] Bialynicki-Birula, I. (1994) On the Wave Function of the Photon. Acta Physica Polonica A, 86, 97-116.

[18] Sipe, J.E. (1995) Photon Wave Functions. Physical Review A, 52, 1875-1883. http://dx.doi.org/10.1103/PhysRevA.52.1875

[19] Wynberg, H., Meijer, E.W., Hummelen, J.C., Dekkers, H.P.J.M., Schippers, P.H. and Carlson, A.D. (1980) Circular Polarization Observed in Bioluminescence. Nature, 286, 641-642. http://dx.doi.org/10.1038/286641a0

[20] Kemp, J.C. and Wolstencroft, R.D. (1972) Interstellar Circular Polarization: Data for Six Stars and the Wavelength Dependence. Astrophysical Journal, 176, L115. http://dx.doi.org/10.1086/181036 